\title{
Propiedades Psicométricas de la Escala de Comunicación Familiar
}

\author{
Psychometric Properties of the Family Communication Scale
}

\author{
Cecilia Cracco $^{1}$ y César Daniel Costa-Ball ${ }^{2}$
}

\begin{abstract}
Resumen
Partiendo del creciente interés transcultural por el Modelo Circumplejo y reconociendo la necesidad de contar con versiones adaptadas de sus escalas, el presente estudio analiza las propiedades psicométricas de la Escala de Comunicación Familiar de Olson y Barnes (2010) en población uruguaya. Se contó con una muestra de 518 familias a la que se administró un cuestionario sociodemográfico, la Escala de Comunicación Familiar y las escalas FACES IV. El análisis factorial confirmatorio corroboró la unidimensionalidad de la escala, con un adecuado índice de fiabilidad, y correlaciones esperables teóricamente con las escalas del FACES con excepción de Enmarañada y Rígida. Se concluye que la Escala de Comunicación Familiar presenta adecuadas propiedades psicométricas que la tornan una técnica válida y fiable para la evaluación de la comunicación en familias uruguayas.
\end{abstract}

Palabras clave: Escala de Comunicación Familiar, análisis factorial confirmatorio, propiedades psicométricas, comunicación familiar, Modelo Circumplejo

\begin{abstract}
Given the growing transcultural interest shown in the Circumplex Model, and considering the need for adapted versions of its scales, the present study analyzes the psychometric properties of the Family Communication Scale (Olson \& Barnes, 2010) in the Uruguayan population. The sample was composed of 518 families. We administered a sociodemographic questionnaire, the Family Communication Scale and FACES IV scales. Results from the confirmatory factor analysis showed the unidimensionality of the scale, presenting an adequate index of reliability. Moreover, the correlations with FACES IV scales agreed with theoretically based expectations, with the exception of the Enmeshment and Rigid scales. We conclude that the Family Communication Scale presents adequate psychometric properties that make it a valid and reliable technique for the assessment of communication in Uruguayan families.
\end{abstract}

Keywords: Family Communication Scale, confirmatory factor analysis, psychometric properties, family communication, Circumplex Model

\footnotetext{
${ }^{1}$ Doctora en Psicología. Directora del Departamento de Psicología Clínica y de la Salud, Universidad Católica del Uruguay. Comandante Braga 2717, CP 11600, Montevideo, Uruguay. Tel.: 598 24872717. Correo: ccracco@ucu.edu.uy

${ }^{2}$ Magíster en Metodología de las Ciencias del Comportamiento y de la Salud. Profesor del Departamento de Neurocognición, Universidad Católica del Uruguay. Comandante Braga 2717, CP 11600, Montevideo, Uruguay. Tel.: 598 24872717. Correo: ccosta@ucu.edu.uy

Revista Iberoamericana de Diagnóstico y Evaluación - e Avaliação Psicológica. RIDEP · N51 Vol.2 · 77-86 · 2019

ISSN: 1135-3848 print /2183-6051online
} 


\section{Introducción}

La Escala de Comunicación Familiar (Olson \& Barnes, 2010) evalúa una variable de especial importancia dentro de la psicología familiar sistémica. Los estudios en torno a la comunicación tomaron protagonismo al explicar las relaciones interpersonales y las dinámicas de las familias desde los primeros desarrollos conceptuales del paradigma (Watzlawick, Beavin, \& Jackson, 1983). Reconociendo su centralidad, Olson y colaboradores, incluyeron la comunicación familiar en el Modelo Circumplejo de los Sistemas Maritales y Familiares (Olson, Sprenkle, \& Russell, 1979).

El Modelo Circumplejo es uno de los modelos que mejor ha logrado articular teoría, clínica e investigación con familias (White \& Klein, 2008), integrando tres dimensiones de gran relevancia para los sistemas maritales y familiares: cohesión, flexibilidad y comunicación (Olson, DeFrain, \& Skogrand, 2014). La cohesión familiar es definida como el lazo emocional que une a los miembros de la familia entre sí (Olson, 2000). Niveles balanceados de cohesión posibilitan un mejor funcionamiento familiar al habilitar tanto la conexión como la independencia de los miembros de la familia. Los extremos en esta dimensión desligados o enmarañados- suelen llevar a relaciones familiares problemáticas si son sostenidos en el tiempo. La flexibilidad familiar es definida como la cantidad de cambio en el liderazgo, roles y reglas familiares (Olson, 2000). Niveles balanceados de flexibilidad llevan a un mejor funcionamiento de la familia. Los extremos de esta dimensión dan cuenta de funcionamientos familiares rígidos o caóticos. La comunicación familiar es considerada la dimensión facilitadora que permite movimientos en las otras dos dimensiones. Los sistemas balanceados se caracterizan habitualmente por poseer muy buenas habilidades de comunicación: empatía, escucha reflexiva, comentarios de apoyo, claridad, coherencia, continuidad, respeto y consideración mutua (Olson, 2000; 2011). El modelo cruza los 5 niveles de las dimensiones de cohesión y flexibilidad familiar para obtener una tipología de 25 tipos de funcionamientos maritales o familiares (Olson, 2011). Las hipótesis centrales del Modelo Circumplejo afirman que las familias balanceadas tenderán a ser más funcionales que las familias desbalanceadas; que las familias balanceadas presentarán una comunicación más positiva que las familias desbalanceadas; y que las familias balanceadas cambiarán sus niveles de cohesión y flexibilidad como respuesta al estrés a lo largo de las distintas etapas del ciclo vital (Olson, 2000; Olson \& Gorall, 2003; 2006). Estas hipótesis han sido puestas a prueba y confirmadas a través de los resultados de cientos de estudios (Kouneski, 2000).

En el contexto iberoamericano, las escalas de evaluación del modelo (paquete FACES) cuentan con adaptaciones en distintos países (Costa-Ball, González-Tornaría, Del Arca, Masjuan, \& Olson, 2013; dos Santos, Rezende, \& Pimenta, 2017; Martínez-Pampliega, Iraguri, \& Sanz, 2011; Martínez-Pampliega, Merino, Iriarte, \& Olson, 2017; Pereira \& Teixeira, 2012; Schmidt, Barreyro, \& Maglio, 2010; Zegers, Larraín, Polaino-Lorente, Trapp, \& Diez, 2003). Los estudios instrumentales de adaptación de técnicas reconocen la importancia de considerar aspectos culturales que inciden en la evaluación de las familias y que pueden llevar al clínico o al investigador a conclusiones $\mathrm{y} / \mathrm{o}$ decisiones erróneas a partir de los resultados obtenidos con técnicas que han sido creadas tomando como referencia familias pertenecientes a un marco cultural diferente (Carretero-Dios \& Pérez, 2005), lo que fundamenta la importancia de este estudio.

La Escala de Comunicación Familiar (Family Communication Scale, FCS) fue creada por Barnes y Olson en 1982 (Barnes \& Olson, 1989). El desarrollo inicial del instrumento se focalizaba en la etapa de la adolescencia, buscando describir la comunicación entre padres e hijos adolescentes (Barnes \& Olson, 1985). En la construcción inicial de la escala, los autores quisieron captar aspectos tales como la apertura o libertad para intercambiar ideas e intereses, la confianza u honestidad experimentada y el tono emocional de las interacciones. De un banco inicial de ítems producto de la revisión de la literatura disponible, seleccionaron 35 para la realización de un primer estudio piloto que permitió reducir la cantidad de reactivos a 20 que se agrupaban en dos subescalas: apertura a la comunicación familiar y problemas en la comunicación familiar (Barnes \& Olson, 1989). Posteriormente, la escala fue 
revisada y reducida a 10 ítems que contemplan solamente las habilidades positivas de la comunicación (mensajes claros y congruentes, empatía, frases de apoyo, habilidades efectivas de resolución de problemas) entre todos los miembros del sistema familiar, en cualquier etapa de su ciclo evolutivo (Olson \& Barnes, 2010). Esta versión de la escala ha sido adaptada a distintos países y poblaciones y es ampliamente utilizada en investigaciones basadas en el Modelo Circumplejo. Entre los estudios más recientes pueden mencionarse los de Martínez-Pampliega et al. (2017), Šeboková y Popelková (2016), Zuñeda, Llamazares, Marañón, y Vázquez (2016), Wershler y Ronis (2015), Koutra et al. (2014), Margasinski (2014), Baiocco et al. (2013), Lehana, Flores, Arango-Lasprilla, Díaz y Espinosa (2012), Pereira y Teixeira (2012). En América Latina se han realizado estudios de adaptación de la escala en Perú (Copez-Lonzoy, Villarreal-Zegarra, \& Paz-Jesús, 2016) y en Chile (Rivadeneira \& López, 2017).

En Uruguay, el trabajo de adaptación de la Escala de Comunicación Familiar se inició en el año 2007, con autorización del Dr. David Olson. En las fases iniciales del proceso de adaptación se realizó la traducción y traducción inversa siguiendo las directrices recomendadas para la adaptación de escalas (Balluerka, Gorostiaga, Alonso-Arbiol, \& Haranburu, 2007; Muñiz, Elousa, \& Hambleton, 2013). Dentro de los estudios realizados para la adaptación de las Escalas FACES IV, se analizaron las propiedades psicométricas primarias de la Escala de Comunicación Familiar, obteniendo un índice de fiabilidad $\alpha$ de Cronbach de .88 (Costa-Ball et al., 2009). El objetivo de este trabajo es completar el proceso de adaptación de la técnica realizando análisis de dimensionalidad, validez y confiabilidad.

\section{Método}

\section{Participantes}

Participaron un total de 518 familias de población general, residentes en la ciudad de Montevideo. La muestra, seleccionada por conveniencia, se tomó a través de distintas instituciones de educación privada formal y no formal. Se marcó como criterio de inclusión que la familia tuviera al menos un hijo dentro del núcleo familiar, conformándose la muestra con familias en distintas etapas del ciclo vital. Se intentó lograr una muestra que reflejara lo más fielmente posible la distribución de los hogares en los distintos niveles socioeconómicos de Montevideo. Las familias participantes tienen en promedio una cantidad de 3.9 integrantes $(D E=1.3)$ con una cantidad de hijos $M=2.1$ $(D E=1.3)$. En la Tabla 1 se presentan datos descriptivos respecto a la configuración de las familias, etapa del ciclo vital, y nivel socioeconómico.

Tabla 1. Características sociodemográficas de las familias participantes en porcentajes

\begin{tabular}{lc}
\hline Configuración familiar & \\
\hline Nuclear & 63.3 \\
Ensamblada & 8.3 \\
Extensa & 11 \\
Monoparental & 16.4 \\
Otros & 1 \\
Etapa de ciclo vital & \\
Con hijos preescolares-escolares (1 a 12 & 56 \\
años) & 23.8 \\
Con hijos adolescentes (13 a 18 años) & 20.2 \\
Con hijos en edad de emancipación (19 & \\
años o más) & \\
Nivel socioeconómico & 15.2 \\
Bajo & 60.7 \\
Medio & 24.1 \\
Alto & \\
\hline
\end{tabular}

\section{Instrumentos}

Cuestionario sociodemográfico e Índice de Nivel Socioeconómico. Con el fin de lograr una descripción de las familias participantes, se elaboró un cuestionario ad hoc en el que se solicitó información sobre la conformación de la familia: configuración, cantidad de integrantes, edades de los hijos. Para evaluar el nivel socioeconómico de las familias se utilizó el Índice de Nivel Socioeconómico (INSE) en su versión reducida (Llambí \& Piñeyro, 2012).

Escala de Comunicación Familiar (Olson \& Barnes, 2010). La escala se compone de 10 ítems que evalúan el intercambio de información, ideas, pensamientos y sentimientos entre los miembros de la familia, pudiendo variar de muy pobre a muy efectiva. Los ítems deben responderse en una escala Likert de 5 puntos donde 1 significa "totalmente en desacuerdo" y 5 "totalmente de acuerdo", siendo la puntuación mínima de la 
escala 10 puntos y la máxima 50 puntos. Puntajes más altos indican mejor comunicación familiar. El índice de confiabilidad $\alpha$ de Cronbach de la escala es .90 , y el índice de correlación test re-test .86, con datos de una muestra norteamericana $N=2465$ (Olson \& Barnes, 2010).

Escalas de Evaluación de la Adaptabilidad y Cohesión Familiar FACES IV (Olson, 2010). FACES IV se compone de seis escalas: cohesión y flexibilidad balanceadas, cohesión desligada y enmarañada, y flexibilidad rígida y caótica (Olson, 2010). Los estudios de validación realizados con la técnica confirman la estructura de 6 factores (índices de ajuste: $\chi^{2}=2,058.76$ $(d f=804, \quad p<.001), \quad$ IFI $=.97, \quad$ CFI $=.97$, RMSEA=.058) (Olson, 2011). Los análisis de correlaciones entre las escalas mostraron que Cohesión correlaciona negativamente con Desligada (-.80) y con Enmarañada, aunque a un nivel muy bajo (-.15). La escala de Flexibilidad correlaciona negativamente con la escala Caótica (-.53) y muy débilmente con la escala Rígida (.12). Los coeficientes de fiabilildad $\alpha$ de Cronbach reportados por Olson (2011) son: .77 para Enmarañada, .87 para Desligada, .89 para Cohesión, .86 para Caótica, .82 para Rígida, y .84 para Flexibilidad. En este trabajo se utilizó la versión abreviada, adaptada a población montevideana por Costa-Ball et al. (2013) en la que cada una de las escalas se compone por cuatro ítems que se puntúan en una escala Likert de 5 puntos donde 1 significa "totalmente en desacuerdo" y 5 significa "totalmente de acuerdo". En las dos escalas balanceadas, puntuaciones más altas indican un funcionamiento familiar más saludable; en las cuatro escalas desbalanceadas, puntuaciones más altas indican un funcionamiento familiar más problemático. Esta versión presentó un buen ajuste al modelo de 6 factores $\left(\chi^{2}(237, N=294)=318.45, p=.000\right.$; $\chi^{2} / g l=1.34 ;$ RMSEA=.034; IFI=.92; CFI=.92; TLI=.91), con índices de consistencia interna aceptables $(\alpha$ de Cronbach=.71 para Cohesión, .60 para Enmarañada, .56 para Desligada, .46 para Flexibilidad, .65 para Caótica, y .57 para la escala Rígida) (Costa-Ball et al., 2013).

\section{Procedimiento}

Para conformar la muestra se contactaron instituciones de educación privada formal y no formal ubicadas en distintos barrios de la ciudad de Montevideo. Se asistió a encuentros con padres organizados por las instituciones al término de los cuales se presentó el trabajo de investigación y se solicitó la participación voluntaria de uno de los adultos responsables de cada familia. Todos los instrumentos utilizados son auto-administrados. El procedimiento, consentimientos y protocolos cuentan con el aval del Comité de Ética de la Facultad de Psicología de la Universidad Católica del Uruguay, cumpliendo las normativas del país sobre investigación con seres humanos reguladas por el Decreto 001-4573/2007 del Poder Ejecutivo y de la Ley Nro. 18331 de Habeas Data, referida a la reserva de datos personales.

\section{Diseño y análisis de datos}

Se trató de un estudio de tipo instrumental (Ato, López, \& Benavente, 2013; Montero \& León, 2007) en el que se realizaron análisis de ítems, dimensionalidad, fiabilidad y validez de la Escala de Comunicación Familiar (Olson \& Barnes, 2010). Se ejecutó un análisis factorial confirmatorio para conocer el grado de ajuste entre los datos empíricos obtenidos y el modelo teórico. Se eliminaron todos los casos en los que había datos perdidos (28 casos). Para tomar las decisiones respecto al método de estimación a utilizar se consideraron el tamaño de la muestra, y el tipo y la distribución de las variables (Ferrando $\&$ Anguiano-Carrasco, 2010). Dado valores altos de asimetría y curtosis (que exceden el rango [-1, 1]), se utilizaron matrices de datos policóricas y el método de estimación robusta de mínimos cuadrados no ponderados (ULS) (Carretero-Dios \& Pérez, 2005; 2007; Lloret-Segura, FerreresTraver, Hernández-Baeza, \& Tomás-Marco, 2014). Se consideraron los índices de ajuste: razón del $\chi^{2}$ sobre grados de libertad, RMSEA (error cuadrático medio de aproximación), CFI (índice de ajuste comparativo), y TLI (índice de ajuste no normado de Tucker-Lewis). Los puntos de corte que dan cuenta de ajuste de los datos al modelo propuesto son: $\chi^{2} / g l<$ a 3; RMSEA < a .08 ; CFI $\geq .95$; TLI $\geq .95$ (Schreiber, Nora, Stage, Barlow, \& King, 2006). Se analizó la fiabilidad o consistencia interna de la escala con el índice $\alpha$ ordinal (Elousa \& Zumbo, 2008; Zumbo, Gadermann, \& Zeisser, 2007). Se realizaron cálculos descriptivos y comparativos utilizando el 
Tabla 2. Medias, desvíos, asimetría, curtosis y correlación ítem-test de la

Escala de Comunicación Familiar

\begin{tabular}{lccccc}
\hline & $M$ & $D E$ & Asimetría & Curtosis & $\begin{array}{c}\text { Correlación ítem-test } \\
\text { corregida }\end{array}$ \\
\hline Ítem 1 & 4.04 & 1.02 & -1.296 & 1.499 & .64 \\
Ítem 2 & 4.09 & 0.95 & -1.451 & 2.313 & .70 \\
Ítem 3 & 4.56 & 0.81 & -2.439 & 6.644 & .72 \\
Ítem 4 & 4.49 & 0.81 & -2.195 & 5.911 & .71 \\
Ítem 5 & 3.91 & 1.09 & -1.064 & 0.508 & .58 \\
Ítem 6 & 4.17 & 0.99 & -1.489 & 2.120 & .56 \\
Ítem 7 & 4.46 & 0.85 & -2.227 & 5.869 & .58 \\
Ítem 8 & 4.45 & 0.85 & -2.173 & 5.543 & .68 \\
Ítem 9 & 3.31 & 1.29 & -0.418 & -0.952 & .34 \\
Ítem 10 & 4.46 & 0.87 & -2.159 & 5.107 & .66 \\
\hline
\end{tabular}

estadístico $H$ de Kruskal-Wallis entre más de dos muestras independientes y la prueba $U$ de MannWhitney acompañada de la corrección de Bonferroni. Los análisis reportados fueron realizados con los programas Statistical Package for the Social Sciences (SPSS, versión 18) y Mplus (versión 7.4).

\section{Resultados}

Para comenzar el análisis factorial confirmatorio de la escala, se llevó adelante el estudio descriptivo de los ítems y se analizó asimetría, curtosis y coeficiente de correlación (ver Tabla 2). Los valores reportados en la Tabla 2 dan cuenta de la necesidad de utilizar matrices policóricas, con el método de estimación de mínimos cuadrados no ponderados (ULS) (Ferrando \& Lorenzo-Seva, 2014).

Los resultados de los indicadores de bondad de ajuste muestran un muy buen ajuste a la estructura unidimensional planteada por Olson y Barnes (2010) para la escala $\left(\chi^{2}(35, N=518)=\right.$ $157.45, p=.000 ; \chi^{2} / g l=4.21 ;$ RMSEA $=.082,90 \%$ IC $[.069, .095]$; CFI=.97; TLI=.96).

En la Tabla 3 se presentan las cargas factoriales y comunalidad de los ítems. Todos los ítems presentan cargas factoriales adecuadas. Se estimó la fiabilidad de la escala, obteniendo un índice $\alpha$ ordinal de .92 .

Con el objetivo de obtener evidencias de validez externa, se calcularon las correlaciones con las escalas FACES IV. Según el modelo teórico, se espera que la Comunicación familiar presente correlaciones significativas y en sentido positivo con las escalas balanceadas de Cohesión y Flexibilidad, y correlaciones significativas y
Tabla 3. Carga factorial y comunalidad de los ítems de la Escala de Comunicación Familiar

\begin{tabular}{lcc}
\hline & Carga factorial & Comunalidad \\
\hline Ítem 1 & .77 & .59 \\
Ítem 2 & .78 & .61 \\
Ítem 3 & .87 & .76 \\
Ítem 4 & .84 & .71 \\
Ítem 5 & .67 & .45 \\
Ítem 6 & .68 & .47 \\
Ítem 7 & .69 & .47 \\
Ítem 8 & .81 & .65 \\
Ítem 9 & .43 & .18 \\
Ítem 10 & .79 & .63 \\
\hline
\end{tabular}

negativas con las escalas: Enmarañada, Desligada, Caótica y Rígida. Los valores reportados en la Tabla 4 muestran que la Comunicación Familiar correlaciona con Cohesión, Flexibilidad, Desligada y Caótica, en el sentido esperado. Aunque con valores más débiles, llama la atención la correlación significativa y positiva entre Comunicación y las variables Enmarañada (.13) y Rígida (.13).

Tabla 4. Correlación entre Comunicación familiar y escalas del FACES IV

\begin{tabular}{lc}
\hline & Comunicación \\
\hline Cohesión & $.61 * *$ \\
Flexibilidad & $.50 * *$ \\
Desligada & $-.48^{* *}$ \\
Enmarañada & $.13 * *$ \\
Rígida & $.13^{* *}$ \\
Caótica & $-.36^{* *}$ \\
\hline$* * p<.01$ &
\end{tabular}

Finalmente, se realizaron análisis descriptivos y comparativos para conocer los puntajes de comunicación familiar obtenidos por las familias participantes, tomando en cuenta su configuración, etapa del ciclo vital, y nivel socioeconómico (ver Tabla 5). 
Tabla 5. Datos descriptivos de la comunicación familiar considerando configuración, etapa del ciclo vital y nivel socioeconómico de las familias

\begin{tabular}{lcc}
\hline Configuración familiar & $M$ & $D E$ \\
\hline Nuclear & 42.7 & 5.4 \\
Ensamblada & 41.5 & 7 \\
Extensa & 40.6 & 7.2 \\
Monoparental & 40.4 & 8.5 \\
$\quad$ Etapa de ciclo vital & & \\
Con hijos prescolares-escolares (1 a 12 años) & 42.7 & 9.2 \\
Con hijos adolescentes (13 a 18 años) & 41.6 & 6.2 \\
Con hijos en edad de emancipación (19 años o más) & 40.4 & 7.7 \\
$\quad$ Nivel socioeconómico & & \\
Bajo & 37.9 & 10.4 \\
Medio & 42.7 & 5.6 \\
Alto & 42.4 & 4.7 \\
\hline
\end{tabular}

En cuanto al tipo de configuración familiar, los resultados de la prueba $H$ de Kruskal-Wallis $\left(\chi^{2}=6.14, g l=3, p<.10\right)$, muestran que no se puede rechazar la hipótesis nula de igual de medias y que debe concluirse que las familias nucleares, ensambladas, extensas y monoparentales, no difieren en sus niveles de comunicación familiar. Respecto al nivel de comunicación familiar en las distintas etapas del ciclo vital, los datos de la prueba $H$ de Kruskal-Wallis $\left(\chi^{2}=10.26, g l=2\right.$, $p<.006)$, muestran que existen diferencias entre las familias con hijos en edad preescolar-escolar, adolescentes, y en edad de emancipación. De las posibles comparaciones entre los grupos, los datos de la prueba $U$ de Mann-Whitney permiten afirmar que las familias con hijos pre-escolares y escolares presentan niveles de comunicación familiar significativamente mayores que las familias con hijos en edad de emancipación ( $U=11079.50 ; p<.004)$. También se encontraron diferencias significativas en los niveles de comunicación familiar reportados por las familias pertenecientes a distintos niveles socioeconómicos $\left(\chi^{2}=15.2, g l=2, p<.001\right)$. Los datos de la prueba de contraste $U$ de Mann-Whitney entre los distintos grupos, utilizando la corrección de Bonferroni, mostraron que entre las familias de nivel socioeconómico medio y alto no existen diferencias significativas en cuanto a la comunicación familiar $(U=17766.50 ; p<.12)$. Sí existen diferencias significativas entre las familias de nivel socioeconómico alto y bajo ( $U=3915.50$; $p<.013)$ y entre los niveles medio y bajo $(U=9017.00 ; p<.000)$, mostrando que son las familias de nivel socioeconómico bajo las que reportan menores niveles de comunicación familiar.

\section{Discusión}

El objetivo de este trabajo fue estudiar las propiedades psicométricas de la Escala de Comunicación Familiar (Olson \& Barnes, 2010), lo cual supone un paso más en la adaptación del Paquete FACES IV para Uruguay. El esfuerzo por lograr la adaptación de técnicas reconoce las diferencias que pueden existir entre las distintas culturas en relación a variables de tipo familiar.

El paquete FACES IV es una de las baterías más utilizadas mundialmente para la evaluación de las familias (García-Méndez, Rivera-Aragón, Reyes-Lagunes, \& Díaz-Loving, 2006; Kouneski, 2000). Estas escalas permiten la evaluación de las dimensiones de cohesión, flexibilidad y comunicación familiar, variables centrales del Modelo Circumplejo de los Sistemas Maritales y Familiares, que han mostrado poseer gran relevancia para el estudio y la intervención con familias (Olson et al., 2014; Walsh, 2012a). Como parte del paquete FACES IV, la Escala de Comunicación Familiar de Olson y Barnes (2010) es uno de los instrumentos más utilizados para la evaluación de la comunicación en los sistemas familiares.

En Uruguay, los trabajos realizados por Costa-Ball y colaboradores (Costa-Ball et al., 2009; Costa-Ball et al., 2013) llevaron a la creación de una versión abreviada de las escalas FACES IV de 24 ítems. Esta versión presenta adecuadas propiedades psicométricas, con valores más débiles para las escalas Enmarañada y Rígida. Otros estudios de adaptación también han reportado dificultades con estas escalas (Pereira \& Texeira, 2012; Rivero, Martínez-Pampliega, \& Olson, 2010). El autor original de la técnica ha 
señalado que estas dos escalas resultan más débiles empíricamente y requieren de mayor estudio y análisis (Olson, 2011).

Respecto a la Escala de Comunicación Familiar (Olson \& Barnes, 2010), los resultados del análisis factorial confirmatorio realizado apoyan la solución unidimensional, con alto índice de fiabilidad. Los estudios de validez externa efectuados con las escalas FACES IV ofrecen resultados coherentes con el modelo teórico subyacente. En este sentido, la comunicación familiar correlaciona en forma positiva con las escalas de Cohesión y Flexibilidad balanceadas, y en forma negativa con las escalas de Desligada y Caótica, dando apoyo a la hipótesis del modelo teórico que afirma que las familias balanceadas presentarán una comunicación más positiva que las familias desbalanceadas (Olson, 2000; Olson \& Gorall, 2003; 2006). Los resultados de las correlaciones entre Comunicación y las escalas Enmarañada y Rígida, fueron significativas también, pero en sentido distinto al esperado. Según los datos, la comunicación se asociaría en sentido positivo al funcionamiento enmarañado y rígido en las familias uruguayas participantes en este estudio. Estos resultados merecen atención y requieren de nuevos trabajos que implicarán seguramente la revisión de la validez de contenido de las escalas Enmarañada y Rígida. Olson (2000) señalaba la importancia de incluir distintas perspectivas en la evaluación del funcionamiento familiar considerando el reporte de la familia, pero también la observación del clínico, en una evaluación multi-método, multi-rasgo y multisistema. La revisión de las escalas Enmarañada y Rígida permitiría dotar de mayor potencia diagnóstica al Paquete FACES IV y habilitaría nuevos análisis respecto a su correlación con la comunicación familiar.

Al analizar los resultados obtenidos con la Escala de Comunicación Familiar en función de las características sociodemográficas de la muestra, no se encontraron diferencias en el nivel de comunicación de las familias según su tipo de configuración familiar. Este resultado es consistente con la idea de que la comunicación familiar no dependerá de cómo se integre la familia, sino de sus patrones de interacción en el tiempo (Walsh, 2012b). En cuanto a las etapas del ciclo vital, fueron las familias con hijos en edad de emancipación las que reportaron menores niveles de comunicación familiar, aspecto que ha sido señalado reiteradamente desde el estudio pionero realizado por Olson y colaboradores en la década de los 80 con familias norteamericanas de población general en distintas etapas del ciclo vital (Olson et al., 1983). Por último, las familias de nivel socioeconómico bajo reportaron niveles significativamente menores de comunicación familiar que las familias en los niveles medio y alto. Los autores que profundizaron en el conocimiento de las familias en situación de pobreza señalan como rasgo distintivo las dificultades en la comunicación en estos sistemas familiares (Cancrini, De Gregorio, \& Nocerino, 1997; Gómez, Muñoz, \& Haz, 2007; Neto, 1996).

Estos análisis muestran la posibilidad de utilizar la Escala de Comunicación Familiar para profundizar en el conocimiento de las características que presentan las familias que se encuentran en distintos contextos y momentos de su ciclo evolutivo. De todas formas, es importante tomar con cautela estos resultados, considerando que la muestra fue tomada por conveniencia.

Podemos concluir este trabajo afirmando que la Escala de Comunicación Familiar de 10 ítems de Olson y Barnes (2010) presenta adecuadas propiedades psicométricas que la tornan una técnica válida y fiable para la evaluación de la comunicación en las familias uruguayas. El estudio presentado en este artículo resulta un aporte a la adaptación y creación de técnicas y baterías de evaluación del funcionamiento familiar en Uruguay y el contexto iberoamericano.

\section{Referencias}

Ato, M., López, J., \& Benavente, A. (2013). Un sistema de clasificación de los diseños de investigación en psicología. Anales de Psicología, 29(3), 1038-1059. doi:10.6018/analesps.29.3.178511

Baiocco, R., Cacioppo, M., Laghi, F., \& Tafà, M. (2013). Factorial and construct validity of FACES IV among Italian adolescents. Journal of Child and Family Studies, 22, 962-970. doi:10.1007/s10826-012-9658-1 
Balluerka, N., Gorostiaga, A., Alonso-Arbiol, I., \& Haranburu, M. (2007). La adaptación de instrumentos de medida de unas culturas a otras: una perspectiva práctica. Psicothema, 19(1), 124-133.

Barnes, H., \& Olson, D. (1985). Parent-adolescent communication and the Circumplex Model. Child Development, 56(2), 438-447. doi: $10.2307 / 1129732$

Barnes, H., \& Olson, D. (1989). Comunicación padres-adolescente. En D. Olson, $\mathrm{H}$. McCubbin, H. Barnes, A. Larsen, M. Muxen, \& M. Wilson, Family Inventories, University of Minnesota, Minnesota. Traducción de A. Hernández, Inventarios sobre familia, (pp. 6380). Universidad de Santo Tomás, Bogotá.

Cancrini, L., De Gregorio, F., \& Nocerino, S. (1997). Las familias multi-problemáticas. En M. Coletti, \& J. Linares (Comp.), La intervención sistémica en los servicios sociales ante la familia multi-problemática, (pp. 45-80). Barcelona: Paidós.

Carretero-Dios, H., \& Pérez, C. (2005). Normas para el desarrollo y revisión de estudios instrumentales. International Journal of Clinical and Health Psychology, 5(3), 521551.

Carretero-Dios, H., \& Pérez, C. (2007). Normas para el desarrollo y revisión de estudios instrumentales: Consideraciones sobre la selección de tests en la investigación psicológica. International Journal of Clinical and Health Psychology, 7(3), 863-882.

Copez-Lonzoy, A., Villareal-Zegarra, D., \& PazJesús, A. (2016). Propiedades psicométricas de la Escala de Comunicación Familiar en estudiantes universitarios. Revista Costarricense de Psicología, 35(1), 31-46.

Costa-Ball, D., González-Tornaría, M., Del Arca, D., Masjuan, N., \& Olson, D. (2013). Propiedades psicométricas del FACES IV: Estudio de validez en población uruguaya. Ciencias Psicológicas, 7(2), 119-132.

Costa-Ball, D., González-Tornaría, M., Masjuan, N., Trápaga, M., Del Arca, D., Scafarelli, L., \& Feibuscheurez, A. (2009). Escala de evaluación del funcionamiento familiar FACES IV-: Proceso de adaptación a Montevideo, Uruguay. Ciencias Psicológicas, 3(1), 43-56. dos Santos, Rezende, \& Pimenta (2017). Family Adaptability and Cohesion Evaluation Scale IV (FACES IV) - adaptação brasileira. Revista Avaliação Psicológica, 16(2), 120127. doi:10.15689/ap.2017.1602.01

Elousa, P., \& Zumbo, B. (2008). Coeficientes de fiabilidad para escalas de respuesta categórica ordenada. Psicothema, 20(4), 896-901.

Ferrando, P., \& Anguiano-Carrasco, C. (2010). El análisis factorial como técnica de investigación en psicología. Papeles del Psicólogo, 31(1), 18-33.

Ferrando, P., \& Lorenzo-Seva, U. (2014). El análisis factorial exploratorio de los ítems: algunas consideraciones adicionales. Anales de Psicología, 30(3), 1170-1175. doi:10.6018/analesps.30.3.199991

García-Méndez, M., Rivera-Aragón, S., ReyesLagunes, I., \& Díaz-Loving, R. (2006). Construcción de una escala de funcionamiento familiar. Revista Iberoamericana de Diagnóstico y Evaluación - e Avaliação Psicológica, 22(2), 91-110.

Gómez, E., Muñoz, M., \& Haz, A. (2007). Familias multiproblemáticas y en riesgo social: características e intervención. Psykhe, 16(2), 43-54. doi:10.4067/s0718-22282007000200004

Kouneski, E. (2000). Family assessment and the Circumplex Model: New research developments and applications. University of Minnesota, Minnesota, United States. Recuperado de http://citeseerx.ist.psu.edu/viewdoc/download ?doi=10.1.1.195.3412\&rep=rep1\&type $=$ pdf

Koutra, K., Triliva, S., Roumeliotaki, T., Stefanakis, Z., Basta, M., Lionis, C., \& Vgontzas, A. (2014). Family functioning in families of first-episode psychosis patients as compared to chronic mentally ill patients and healthy controls. Psychiatry Research, 219, 486-496. doi:10.1016/j.psychres.2014.06.045

Lehana, T., Flores, L., Arango-Lasprilla, J.C., Díaz, D., \& Espinosa, I. (2012). Balancing act: The influence of adaptability and cohesion on satisfaction and communication in families facing TBI in Mexico. NeuroRehabilitation, 30, 75-86. doi:10.3233/NRE-2012-0729 
Llambí, C., \& Piñeyro, L. (2012). Índice de nivel socioeconómico (INSE). Centro de Investigaciones Económicas, Montevideo, Uruguay.

Lloret-Segura, S., Ferreres-Traver, A., HernándezBaeza, A., \& Tomás-Marco, I. (2014). El análisis factorial exploratorio de los ítems: Una guía práctica, revisada y actualizada. Anales de Psicología, 30(3), 1151-1169. doi:10.6018/analesps.30.3.199361

Margasinski, A. (2014). An outcome study of alcoholic families in Poland using FACES IV. Journal of Family Psychotherapy, 25, 348358. doi:10.1080/08975353.2014.977684

Martínez-Pampliega, A., Iraguri, I., \& Sanz, M. (2011). Validez estructural del FACES20Esp: Versión española de 20 ítems de la Escala de Evaluación de la Cohesión y Adaptabilidad Familiar. Revista Iberoamericana de Diagnóstico y Evaluación - e Avaliação Psicológica, 29(1), 147-165.

Martínez-Pampliega, A., Merino, L., Iriarte, L., \& Olson, D. (2017). Psychometric properties of the spanish version of the Family Adaptability and Cohesion Evaluation Scale IV. Psicothema, 29(3), 414-420. doi:10.7334/psicothema2016.21

Montero, I., \& León, O. (2007). A guide for naming research studies in Psychology. International Journal of Clinical and Health Psychology, 7(3), 847-862.

Muñiz, J., Elosua, P., \& Hambleton, R. (2013). Directrices para la traducción y adaptación de los tests: segunda edición. Psicothema, 25(2), 151-157. doi:10.7334/psicothema2013.24

Neto, L. (1996). Familias pobres y multiasistidas. En M. Millán (Comp.), Psicología de la familia. Un enfoque evolutivo y sistémico ( $\mathrm{pp}$. 201-227). Valencia: Promolibro.

Olson, D. (2000). Circumplex Model of Marital and Family Systems. Journal of Family Therapy, 22, 144-167. doi:10.1111/1467-6427.00144

Olson, D. (2010). FACES-IV Manual. Minneapolis: Life Innovations.

Olson, D. (2011). FACES IV and the Circumplex Model: Validation study. Journal of Marital and Family Therapy, 37(1), 64-80.

doi:10.1111/j.1752-0606.2009.00175.x
Olson, D., \& Barnes, H. (2010). Family Communication Scale. Minneapolis, MN: Life Innovations, Inc.

Olson, D., DeFrain, J., \& Skogrand, L. (2014). Marriages and families. Intimacy, diversity and strengths $\left(8^{\text {th }}\right.$ ed.). New York: McGraw Hill.

Olson, D. \& Gorall, D. (2003). Circumplex Model of Marital and Family Systems. En F. Walsh (Ed.), Normal Family Processes ( ${ }^{\text {er }}$ ed.) (pp. 514-547). New York: Guilford Press.

Olson, D., \& Gorall, D. (2006). FACES IV \& the Circumplex Model. Minneapolis, MN: Life Innovations, Inc.

Olson, D., McCubbin, H., Barnes, H., Larsen, A., Muxen, M., \& Wilson, M. (1989). Families: What makes them work. ( $2^{\text {nd }}$ ed.). California: Sage.

Olson, D., Sprenkle, D., \& Russell, C. (1979). Circumplex Model of Marital and Family System: I. Cohesion and adaptability dimensions, family types, and clinical applications. Family Process, 18(1), 3-28. doi:10.1111/j.1545-5300.1979.00003.x

Pereira, M., \& Teixeira, R. (2012). Portuguese Validation of FACES-IV in adult children caregivers facing parental cancer. Contemporary Family Therapy, 35, 478-490. doi:10.1007/s10591-012-9216-4

Rivadeneira, J., \& López, M. A. (2017). Escala de Comunicación Familiar: validación en población adulta chilena. Acta Colombiana de Psicología, 20(2), 116-126. doi:10.14718/ACP.2017.20.2.6

Rivero, N., Martínez-Pampliega, \& Olson, D. (2010). Spanish adaptation of the FACES IV questionnaire: Psychometric characteristics. The Family Journal: Counseling and Therapy for Couples and Families, 18(3), 288-296. doi:10.1177/1066480710372084

Šeboková, G., \& Popelková, M. (2016). Selfconsciousness and internalizing problems in adolescence: Moderating effect of family variables. Studia Psychologica, 58(2), 105121. doi:10.21909/sp.2016.02.710

Schmidt, V., Barreyro, J. \& Maglio, A. (2010). Escala de evaluación del funcionamiento familiar FACES III: ¿Modelo de dos o tres factores? Escritos de Psicología, 3(2), 30-36. 
Schreiber, J., Nora, A., Stage, F., Barlow, E., \& King, J. (2006). Reporting structural equation modeling and confirmatory factor analysis results: a review. The Journal of Educational Research, 99(6), 323-338. doi:10.3200/JOER.99.6.323-338

Walsh, F. (2012a). Family resilience. Strengths forged through adversity. En F. Walsh (Ed.), Normal family processes. Growing diversity and complexity (4 ${ }^{\text {th }}$ ed.), (pp. 399-427). New York: The Guilford Press.

Walsh, F. (2012b). The new normal. Diversity and complexity in $21^{\text {st }}$-century families. En F. Walsh (Ed.), Normal family processes. Growing diversity and complexity ( $\left.4^{\text {th }} \mathrm{ed}.\right)$, (pp. 3-27). New York: The Guilford Press.

Watzlawick, P., Beavin, J., \& Jackson, D. (1983). Teoría de la comunicación humana ( ${ }^{\text {ra }}$ ed.). Barcelona: Herder.

Wershler, J., \& Ronis, S. (2015). Psychosocial characteristics and service needs of Canadian suburban male youth at risk for homelessness. Children and Youth Services Review, 55, 2936. doi:10.1016/j.childyouth.2015.05.009 0

White, J., \& Klein, D. (2008). Family theories ( $3^{\text {rd }}$ ed.). California: Sage.

Zegers, B., Larraín, M., Polaino-Lorente, A., Trapp, A., \& Diez, I. (2003). Validez y confiabilidad de la versión española de la Escala de Cohesión y Adaptabilidad Familiar (CAF) de Olson, Russell \& Sprenkle para el diagnóstico del funcionamiento familiar en la población chilena. Revista Chilena de NeuroPsiquiatría, 41(1), 39-54. doi:10.4067/s0717-92272003000100006

Zumbo, B. D., Gadermann, A. M., \& Zeisser, C. (2007). Ordinal versions of coefficients alpha and theta for Likert rating scales. Journal of Modern Applied Statistical Methods, 6(1), 2129. doi:10.22237/jmasm/1177992180

Zuñeda, A., Llamazares, A., Marañón, D., \& Vázquez, G. (2016). Características individuales y familiares de los adolescentes inmersos en violencia filio-parental: La agresividad física, la cohesión familiar y el conflicto interparental como variables explicativas. Revista de Psicopatología y Psicología Clínica, 21(1), 21-33. doi:10.5944/rppc.vol.21.num.1.2016.15021 